\title{
The network structure of knowledge sharing among physicians
}

\author{
Paola Zappa
}

Published online: 26 March 2011

(C) Springer Science+Business Media B.V. 2011

\begin{abstract}
This paper applies social network analysis in order to model knowledge sharing among hospital physicians. Drawing on the literature on the diffusion of innovation and cooperation in clinical settings, it aims to furnish better understanding of knowledge sharing in two directions: describing how knowledge flows and identifying individual and contextual factors which facilitate its spontaneous spreading. Used to address these issues is a linktracing sample of about 800 Italian hospital physicians, potentially involved in prescribing a new drug. The paper represents knowledge sharing about the innovation as a network. It therefore specifies Exponential Random Graphs (ERG) or $p^{*}$ models to reconstruct the network structure of knowledge sharing and to test the effect of exogenous factors on the tendency to take action in the network. The results show that knowledge flows informally, exploiting mutual information-seeking relationships, and, consistently with previous studies, locally, with physicians tending to cluster in small groups of proximate and similar peers. Moreover empirical evidence is provided that the propensity to share information with colleagues is greatly affected by individual-specific characteristics, mainly by the experience in the field and the attitude toward the innovation, and by exposure to commercial communication.
\end{abstract}

Keywords Innovation $\cdot$ Healthcare $\cdot$ Knowledge sharing $\cdot$ Social networks · ERG or $p *$ models

\section{Introduction}

In explaining knowledge creation and diffusion, the extant literature has placed great emphasis on the role of social interaction. Through communication, cooperation or specific advice seeking, individuals are expected to gain access to new knowledge and to transfer their own knowledge to others. Moreover they can acquire information on the existence of new products or services, obtain evidence of their benefits or be induced to take up innovations.

P. Zappa $(\varangle)$

Department of Economics, University of Milano-Bicocca, 20126 Milan, Italy

e-mail: paola.zappa1@unimib.it 
Knowledge spread has been extensively investigated in healthcare in particular, and several scholars (Burt 1987; Coleman et al. 1966; Fennell and Warnecke 1988; Nair et al. 2008; Trzeciak et al. 2006; Valente 1996) have shown that it takes place through social networks of physicians. Physicians usually rely on their colleagues as sources of information. Indeed, many of them rate colleagues as their most valued source of information and influence. To cite only a few studies, Keating et al. (2007) claim that physicians rely on their colleagues for information and advice about the care of their patients. Trzeciak et al. (2006) emphasize the importance of social networks in implementing therapeutic support protocols. Finally Kaluzny et al. (1989) highlight their role in disseminating information on state-of-the-art cancer treatment practice to physicians employed in peripheral research centers.

Even more importantly, social networks accelerate the generalized comprehension of innovations. They contribute to reducing the barriers to change and encourage the diffusion of new ideas, technologies or products.

The interest in collaboration and knowledge sharing among physicians has significantly increased in recent years. The coordination of clinical and research activities in regional or inter-regional networks of institutions and highly skilled physicians has been promoted by several National Health Services (hereafter NHSs). Moreover, the opportunity to share knowledge with colleagues has been greatly supported by the recent diffusion of information and communication technology platforms, which have led to the creation of experimental virtual communities of physicians. These are based on the spontaneous contribution of geographically dispersed physicians and assume that especially the most research active and specialized ones are keen to interact with peers, and that this behavior is not affected by geographic distance. Nevertheless, there is still a discrepancy between the scenarios depicted by these projects and the findings in the literature. Most studies suggest, in fact, a preference for local interaction and marked heterogeneity in the decision to share knowledge with others. Especially the latter claim, however, has not been to date clearly supported by strong empirical evidence.

Cooperation in clinical settings has been explored by few organizational studies which have assumed very small and geographically close groups as a unit of analysis. Most papers, by contrast, have been mainly concerned with the effectiveness of social networks. These studies have treated individuals as passive adopters, and assigned them little or no agency in both choosing their discussion partners and up-taking innovations. Scholars, moreover, have devoted scant attention to identifying the structure of a knowledge-sharing network or the physician- or context-specific factors which influence the participation in networking. As a consequence, it is still largely unknown whether a physician network is shaped by a tendency to closure in small, closed and local communities of peers, or to openness, with the power and informational advantage of actors, i.e. opinion leaders, who bridge disconnected actors and provide new knowledge. Similarly, it has not been clearly shown which characteristics make individuals heterogeneous in their propensity to share information.

In an attempt to shed light on these issues, this paper explores knowledge sharing in a sampled group of Italian hospital physicians. The sample is constructed in a way which accounts for the possible existence of interaction among geographically dispersed individuals and controls for the effect of different work environments (i.e. type of hospital, investments in patents and research, number of patients assisted, etc.). Observation is made of how these physicians learn about a new drug and the structure of the knowledge-sharing network is then reconstructed. To verify the existence of heterogeneity in the propensity to share knowledge, we control for the effects of individual and contextual characteristics reported in the literature to possibly affect physicians' choices to take action in the network. 
The paper is structured as follows. Section 2 briefly reviews studies on physician networks and the diffusion of innovation in medical settings. It then highlights some aspects which require further investigation and describes how they will be addressed. Section 3 illustrates the sampling process and the dataset. It also describes the methodology used to examine knowledge sharing, Exponential Random Graphs or $p^{*}$ models (Snijders et al. 2006) for social networks. Section 4 sets out the most significant results. Finally, Sect. 5 concludes.

\section{Theoretical framework}

The most significant contribution to explanation of knowledge sharing in healthcare has been made by the large body of literature on the diffusion of innovations. This points out the existence of a social contagion mechanism, whereby physicians are informed and, sometimes, induced to take up an innovation by their peers.

The existence of this influence mechanism was first posited in a seminal work by Coleman et al. (1966) on the diffusion of a new broad spectrum antibiotic within a large community of GPs. These authors found that socially integrated doctors, defined by the number of ties with other physicians, had learned about the new drug from their peers. They were also more likely to adopt an innovation than socially isolated colleagues. These general findings have been subsequently confirmed by a number of studies which explore specific aspects of social contagion. Burt (1987) attributed this influence to esteem and mainly to competitive concerns. Burt and Janicik (1996) showed that the effect of social contagion is rather small in magnitude. Finally, Valente (1996) found the existence of individual thresholds to innovation adoption. Because these papers focus on the effectiveness of social contagion, they provide only few insights into the structure of a knowledge-sharing network and into the factors which affect the decision to participate in it. Most of these insights can be derived only indirectly. Moreover, they refer mainly to local groups of GPs, which are hardly representative of the medical community.

In regard to the former topic, authors have shown that social contagion takes place mainly through advisorship relationships. They lead to the emergence of opinion leaders (Nair et al. 2008), who adopt the innovation and then transfer their knowledge and beliefs to other physicians, exerting their personal influence on the less active and informed ones. Opinion leaders are individuals who are particularly ready to take up innovations (Katz and Lazarsfeld 1955; Nair et al. 2008). They are also knowledgeable physicians who are research active and benefit from high reputation within the scientific community (Valente and Pumpuang 2007). Opinion leaders display a high likelihood to be chosen as advice-seeking partners and a low propensity to seek advice from others. This suggests the existence of highly centralized networks organized around them. However, scholars have not gone further in attempting to identify whether this knowledge transfer mechanism consists only in dyadic interaction among opinion leaders and others, or whether they are part of a wider and more complex network structure. This opinion leadership is exerted mostly at local level, and peers are relatively proximate to the focal individual both organizationally and geographically (Valente and Davis 1999; Clark et al. 2010).

With reference to the possible sources of heterogeneity among physicians, some papers report the existence of individual and context-specific factors which, together with social interaction, affect adoption choice and timing. The same factors are expected to influence also the propensity to share knowledge or to seek advice from others.

The first category of factors comprises individual characteristics. These make physicians more or less interested in the innovations, autonomous in their choices to uptake them and, 
possibly, also more or less willing to rely on their peers as a source of information. Cohen and Levinthal (1990) and Strang and Tuma (1993), among others, cite experience in the field as a crucial factor. It develops over time through learning by doing, mainly from patient feedback (Coscelli and Shum 2004), and is stated to affect the attitude toward innovation in two rival ways. Lave and Wenger (1991) argue that experience increases the individual ability to understand, evaluate and, eventually, accept the innovation. By contrast, Barlow and Burn (2008) show that age, which is connected with experience, may exert a negative influence on adoption, because older clinicians are usually more resistant to change than younger ones. Furthermore, Hirschman (1980) highlights the importance of physicians research orientation, claiming it could be well representative of their interest in novelty. Research active physicians are expected to develop a positive attitude toward the innovations independently from the external pressure to adopt them.

Other scholars cite the hierarchical position within the peer community, as a factor of heterogeneity. They argue that a high hierarchical position is a considerable barrier to innovation acceptance. Hierarchical position can act as a sort of reputation effect (Gollop et al. 2004), which induces some staff to resist change for fear of losing power or autonomy. Moreover, a higher hierarchical position frequently corresponds to less involvement in clinical practice, and consequently to scant interest in innovation (Van den Bulte and Joshi 2006).

The second category of factors, contextual characteristics, comprises mainly sources of information alternative to interaction with peers, for instance scientific and commercial communication. Studies focus especially on the latter and some scholars (Van den Bulte and Lilien 2001) have demonstrated that it is more effective than knowledge sharing. In regard to the adoption of drugs and devices, these sources consist of marketing activities implemented by pharmaceutical companies (Strang and Tuma 1993; Mukherjee 2002).

Some organizational papers have attempted to model the network structure of knowledge sharing, controlling for the effects of individual heterogeneity. They have examined cooperation in clinical settings and applied either descriptive network measures (density, closeness, betweenness) or stochastic models $\left(p_{2}\right)$. These studies generally find that physicians exhibit a clear propensity to build strong and mutual ties (Keating et al. 2007) based on interpersonal trust, like opinion sharing, advice seeking or informal discussion with colleagues (West et al. 1999). The high reciprocity effect, in particular, suggests that many discussions involve more than unilateral advice and give rise to dense, decentralized and closed networks of peers. Each physician has access to relevant information, and control over their dissemination cannot be exerted.

These papers, moreover, verify that discussion and knowledge sharing is shaped by opportunity-physical proximity - although it is not clear whether interaction boundaries are to be placed at department, hospital or regional level (Fattore and Salvatore 2010). Furthermore, cooperation is highly efficient, because influential physicians are those with the most experience in the field. Finally, interactions are limited in their multidisciplinarity and physicians tend to communicate mainly to individuals within their own professional group (Creswick and Westbrook 2006).

Although extensive and mostly consistent with findings on the diffusion of innovation, also the theoretical framework depicted by organizational studies furnishes only a partial picture of knowledge-sharing networks. The main focus of these studies is on collaboration among physicians in routine activities, not specifically on knowledge sharing or innovation. Nor do they examine heterogeneity in individual behavior due to different attitudes, like those toward innovation. Furthermore, they mostly adopt as their unit of analysis a closed and very local community, for instance a single department or hospital organization, and do not take account of the propensity of physicians to establish relationships with distant colleagues. 
This paper adds to the background just described and seeks to extend it. In detail, it addresses the following two research questions:

- Is the network of knowledge sharing on innovation shaped by a tendency to closure in small groups of peers or to openness in a network structure centralized around opinion leaders?

- Is the choice of engaging in knowledge-sharing affected by the individual and contextual characteristics which influence innovation adoption?

In order to answer these questions, we examine knowledge flow on innovations from a selection perspective. As in organizational studies on cooperation, individual characteristics and attitudes are assumed here to be exogenous predictors of network ties (Robins et al. 2001). Hence, physicians are not conceived as passive receivers of information, but as active actors, who autonomously select their interaction partners and participate in networking. The analysis adopts an ecological and systemic approach to innovation adoption. Drawing on the literature on the diffusion of innovations, the process by which physicians uptake (or fail to uptake) innovations is viewed as a complex combination of different factors. They are the above-mentioned knowledge-sharing and individual and context-specific characteristics. It is therefore assumed that these characteristics are also likely to affect how actively physicians seek out their peers. We then attempt to verify whether and to what extent individual heterogeneity in these characteristics is also predictive of the propensity to share knowledge with others. In detail, we posit that positive attitude toward the innovation, high hierarchical position and greater availability of alternative sources of information negatively affect the propensity to interact with colleagues.

Moreover, the structure of the network is examined in order to identify how knowledge is shared. In particular, we aim to depict whether interaction takes place in an open system, which provides access to new resources and information, or in a closed one, which is shaped by reciprocity and trust.

Furthermore, account is taken of prominence in the field, as well as of the existence and extent of opinion leadership, and of multidisciplinarity and proximity in interaction. Differently from past research, here network boundaries are defined at a national level, the purpose being to verify the existence of relationships with distant colleagues. Finally, chosen as an empirical setting is a large community of specialist hospital physicians. They are expected to differ from the GPs frequently observed in traditional studies on innovation diffusion in respect to two factors: the level of uncertainty faced and the propensity to cooperate with colleagues.

\section{Research design}

\subsection{Data}

\subsubsection{Sample}

This study uses an original dataset of Italian hospital physicians and observes knowledge sharing among them in respect to a specific episode: the decision to adopt a new anti-pain drug.

This represents an ideal setting in which to verify this study's hypotheses. The new drug was specifically targeted on the treatment of post-surgery and terminal cancer patients and could be prescribed only in hospital settings. It was addressed mainly to oncologists and 
anesthetists. These physicians, especially oncologists, are geographically dispersed, because their departments are present only in some hospitals. They also face a high level of uncertainty, because they frequently deal with critical patients or experimental treatments. Furthermore, they are very research active. They are thus likely to have a greater propensity to cooperate and to be connected with each other more than other physicians observed in previous studies.

The most innovative aspects of the new drug consisted in the chemical substance used and in the administering method. Compared to other products of the same therapeutic class already on sale, it thus represented a significant incremental innovation. However, a number of physicians had previous experience of similar drugs and could be aware of its benefits.

The producer, a large multinational pharmaceutical company, launched the new drug on the Italian market at the beginning of 2008 and advertised it with a detailing campaign. The survey was conducted a few months later (spring 2008).

The population of Italian oncologists and anesthetists consists of approximately 10,000 individuals, employed in different types of hospital organizations. It was thus impossible to include them all in our analysis. Hence, this is limited to a sample $S$ of the population $N$.

To build the sample we adopted a multi-wave link-tracing design. ${ }^{1}$ The seeds, $S_{0}$, were selected with a proportional stratified sampling. They were extracted from the list of the Italian oncologists and anesthetists provided by the Italian order of physicians (Fnomceo). We stratified them according to their location (region) and affiliation (hospital organization category). From each stratum we then randomly extracted a subsample of physicians with a sampling fraction proportional to that of the total population. In order to have an adequate representation of each stratum, we imposed an overall sample size $S_{0}$ equal to 200. The 200 sampled physicians were provided with a brief description of the main characteristics of the new drug (for those who had not yet adopted it) and were administered a sociometric questionnaire. Interviews were conducted with CATI. The doctors were asked to name colleagues to whom they went for information. ${ }^{2}$ In order to study opinion leadership, it was also collected whether they thought that those colleagues were particularly knowledgeable in the field. ${ }^{3}$ The number of nominations was not fixed, so as to avoid distortions in results. Each named colleague, $S_{1}$, was administered the same questionnaire (wave 2) and the procedure was repeated until wave 4 . At wave 4 only two new physicians were cited, while most of the physicians named colleagues already in the network. The sampled network was thus almost

\footnotetext{
1 A multi-wave link-tracing design consists of:

- Sampling at random a subset of the actors.

- Observing all dyads involving the selected individuals.

- Identifying all individuals reported to have at least one relation with the initial sample, and selecting them with probability 1 .

- Observing all dyads involving the newly selected individuals.
}

The procedure is repeated till wave $m$ (Handcock and Gile 2010).

2 In detail, they were asked: 'In order to decide whether to prescribe the new drug which colleagues would you go for information on it?' In this way, we did not focus explicitly on advice seeking, but we more generally attempted to collect data on either formal or informal information exchange, i.e. discussion.

3 We assessed this information by asking: 'Which colleagues do you consider as knowledgeable about the new drug?'. It was specified that this kind of opinion leadership did not require interaction, but only following research advancements, workshops, etc. of the cited physician (Zappa and Mariani 2011). Then, respondents were allowed to name as knowledgeable also physicians with whom they did not discuss treatments or diagnoses. Overall the physicians cited as prominent, but not as discussion partners, were 107. For the purposes of this study, we used prominence as an attribute of knowledge-sharing colleagues, and excluded the others from the analysis. 
saturated (Doreian and Woodard 1994). ${ }^{4}$ These two new physicians were included in the sample, and then the sampling procedure was halted.

The final sample consisted of 784 physicians. 711 out of 784 completed the questionnaire, so that the overall response rate was $89.4 \%$. In detail, it was $100 \%$ for wave $1,86.3 \%$ for wave $2,82.1 \%$ for wave 3 and $89.3 \%$ for wave 4 . Also for non-respondents it was possible to collect data on most of the variables described in the next section (specialty, affiliation, etc.). A check showed that non-respondents did not significantly differ from respondents for either these variables or sociodemographic characteristics (gender, age). It was therefore decided not to treat them separately in the analysis.

The network displayed an extremely high and unusual level of reciprocity (93\% of the observed dyads were mutual). As for non-respondents we observed that there was not significant difference in any characteristics between who reciprocated a relationship and who did not. This high value of reciprocity suggested that most of the physicians preferred to discuss about the new drug with their colleagues instead of simply seeking their advice, consistently with Keating et al. (2007). To carry out the analysis, it seemed thus reasonable to symmetrize the network at maximum and treat the relationship as symmetric. In this way, we accounted for knowledge flows in both directions within dyads.

Prominence in the field was represented as an individual covariate. For each physician we computed the number of nominations received and stored them in a new variable. Then, we examined its distribution. Physicians cited as prominent received up to 5 nominations, the average number of citations was well below 1 (mean $=0.58)$ and the standard deviation high (st.dev. $=1.02$ ). In detail, $30 \%$ of the sampled physicians were cited as knowledgeable and only $19.4 \%$ received more than 1 nomination. Although these findings could be biased by the research design, they suggest that very prominent physicians are hardly chosen as discussion partners. Knowledgeable physicians in our sample are regarded as prominent by a very small number of colleagues. This is consistent with the hypothesis of Valente and Davis (1999) and Clark et al. (2010) on local opinion leadership.

\subsubsection{Variables}

We collected data on several individual variables. Some of them are the hypothesized sources of heterogeneity in knowledge sharing on innovations (external communication, research orientation, experience and hierarchical position). The others are control variables which are stated in the literature to shape a cooperation network (specialty, affiliation).

By means of the questionnaire we determined the amount of external communication received on the new drug. This variable was captured as the number of visits from detailing professionals. It is the main source of commercial information, because the advertising of drugs is forbidden by Italian law.

The other variables were obtained from various on line publicly available data sources: the Italian order of physicians website, the Italian NHS website, Pubmed and BiomedExperts. ${ }^{5}$

Research orientation and experience could not be observed directly. We therefore defined a proxy to measure them both. In detail, research orientation was measured as research outcomes, i.e. the number of peer-reviewed publications. ${ }^{6}$ We assumed that the greater the

\footnotetext{
$4 S_{m}$ tending to $\emptyset$ for $m=4$.

5 Pubmed and BiomedExperts store information on the papers published by each physician on international peer reviewed journals during his/her career.

6 Before collecting desk data, we conducted indepth interviews to a small sample of oncologists in order to select an appropriate proxy for research orientation. The number of publications was the most cited variable.
} 
research orientation, the greater the interest in scientific activities, and therefore also the higher the research productivity. We gathered this information from Pubmed and, in case of unclear attribution, we double-checked with BiomedExperts. The effect of publications entered the model as the average annual number of peer-reviewed publications a physician had contributed either as the author or as a member of the research group. Because this variable has a very skewed distribution, we applied to it a logarithmic transformation. Experience was captured by professional age, i.e. the number of years since the doctor had graduated. To account for the shape of the experience curve, which tends to increase at a decreasing marginal rate, the effect of professional age entered the model as a square root function of years.

Hierarchical position was a measure of the tasks assigned to each physician. It was captured by the physician's role in his/her work team. We distinguished between physicians with a honorary position, i.e. heads of departments or units with administrative tasks, and others. Hierarchical position entered the model as a dummy.

In regard to specialty, we collected data on the specific specialty of each sampled physician. We then recoded this information into three categories: oncologists, anesthetists and others. Professional affiliation was the hospital organization to which each physician belonged. We kept the information on the specific organizations and used it in the analysis; we also created a new variable, which represented the hospital category. From more to less research-oriented they are: Research Centers and Foundations, University Hospitals, Hospitals and Local Health Units, Private Organizations. ${ }^{7}$ They differ by the number of patients treated and the beds and specialties available, by the amount of public funding received and, more informatively in this study, by interest in research activities.

The main characteristics of the sample are reported in Table 1 . The physicians interviewed were employed in 338 hospital organizations. Their geographic distribution covered all the 20 Italian regions and a variety of locations. All the categories of hospital organizations were represented within the sample in proportions reflecting their real distribution in the population. The physicians were highly heterogeneous, mainly in the number of publications and in the amount of detailing received on the new drug. In regard to specialty, they were mainly oncologists and anesthetists $(82.50 \%)$. The remaining $17.50 \%$ covered 21 specialties, although the most were experts in anti-pain treatments, thus very similar to oncologists. Hence, most physicians named colleagues in their scientific reference group. The average number of annual publications was low (0.418) and its distribution skewed. The same observations can be done for the visits from detailing professionals. In particular, the high standard deviation value suggests that a number of physicians had not yet be exposed to alternative sources of information when the survey was conducted. Finally, 35.90\% of the physicians was in a honorary position. This is consistent with the professional age, which was on average high (23.270 years). Finally, we observed that youngest physicians were mainly employed in Research Centers and Foundations. We did not find any other significant correlation between the individual variables.

\section{Footnote 6 continued}

Nevertheless, it is worthy to recall that it may be biased. Since heads of departments are frequently formally enrolled in each study carried out by their research team, the number of publications might reflect the physicians hierarchical position more than their research orientation. In our sample, however, the two variables were independent $(\rho=-0.08)$.

7 The Italian NHS distinguishes among eight types of hospital organization. To facilitate interpretation, they were reclassified into four classes. 
Table 1 Descriptive statistics for individual covariates

\begin{tabular}{|c|c|c|c|}
\hline & Observations & Mean (st.dev.) & Percentage \\
\hline Publications per year, average ${ }^{a}$ & 784 & $0.418(1.087)$ & \\
\hline Detailing & 784 & $1.409(2.794)$ & \\
\hline Professional age $^{\mathrm{a}}$ & 771 & $23.270(9.861)$ & \\
\hline \multicolumn{4}{|l|}{ Affiliation } \\
\hline Research centers and foundations & 84 & & 10.80 \\
\hline University hospitals & 101 & & 13.00 \\
\hline Hospitals and local health units & 515 & & 66.40 \\
\hline Private organizations & 76 & & 9.80 \\
\hline \multicolumn{4}{|l|}{ Specialty } \\
\hline Anesthetists & 372 & & 47.40 \\
\hline Oncologists & 275 & & 35.10 \\
\hline Others (21 specialties) & 137 & & 17.50 \\
\hline \multicolumn{4}{|l|}{ Hierarchical position } \\
\hline Honorary position & 270 & & 35.90 \\
\hline Other & 482 & & 64.10 \\
\hline
\end{tabular}

${ }^{\text {a }}$ Mean computed on original values $(n=784)$

\subsection{Methods}

\subsubsection{Model}

We represented knowledge sharing within our sampled community as a binary undirected network $y$ whose nodes were physicians and ties were knowledge flows among couples of them. The network corresponded to a symmetric adjacency matrix of size $n \times n$. Each generic element $y_{i j}$ of the matrix equaled 1 if there was a tie between node $i$ and node $j$ $(i=1, \ldots, n ; j=1, \ldots, n ; i \neq j)$ and 0 otherwise.

We then applied the Exponential Random Graph (ERG) or $p^{*}$ models for social networks (Wasserman and Pattison 1996). The ERGMs is a class of stochastic models which capture the structure of empirical networks, allowing inference to be made about the underlying processes. ERGMs conceive the generic network tie $Y_{i j}$ as a random variable, with $y_{i j}$ as the observed value of $Y_{i j}$. They are based on the assumption that the observed network $y$ is one realization of a set of possible networks $Y$ with a fixed number of nodes $n$, and thus as the outcome of an unknown stochastic process. It is also hypothesized that the probability of observing a specific global network structure depends on the presence of various network configurations, i.e. processes occurring in the local social neighborhoods of the network itself. The strength of these local structural effects is indicated by the model parameters. In detail, the class of ERGMs follows the most general form (Snijders et al. 2006):

$$
P_{\theta}(Y=y)=\exp \left(\theta^{\prime} u(y)-\psi(\theta)\right) \quad y \in Y
$$

(i) $y$ is the network; (ii) $u(y)$ is a $p$-dimensional vector of network statistics; (iii) $\theta$ is a $p$-dimensional parameter; (iv) $\psi(\theta)$ is a function which ensures the normalization requirement that the probabilities sum to 1 . 
The estimation of the parameters is performed via a Monte Carlo Markov chain (MCMC) ML procedure for dyadic dependence models (Snijders 2002). The partial conditional independence assumption (Pattison and Robins 2002) implies, in particular, that whether two generic ties $y_{i j}$ and $y_{u v}$ are independent conditionally on the rest of the network depends not only on whether they share nodes but also on the pattern of ties in the rest of the network, even if they do not directly involve $i$ and $j$. In the MCMCML estimation approximate estimates are refined by comparing the observed networks against a distribution of random networks generated by a stochastic simulation using the approximate parameter values. When convergent estimates are obtained, then simulation from the estimates will produce distributions of networks in which the observed one is typical for all of the effects in the model.

\subsubsection{Effects}

We specified two different sets of effects: structural effects and individual ones. In building the models, we adopted a stepwise method to include the parameters. We selected blocks of similar parameters one by one and tested the goodness of fit increase (the goodness-of-fit tests we used are illustrated in the next section).

In order to model the network structure (Model 1), we first specified density and degree distribution. The latter is measured as alternating $k$-star and captures the tendency to engage in relationships. In this study a positive value of the alternating $k$-star parameter stands for the propensity of physicians to discuss with a number of their colleagues, therefore to have access to multiple sources of information and to transfer their own knowledge to others. Then, we verified the propensity to closure (the second research question), and therefore to share knowledge in small group of peers. In closed communities there is no hierarchy, each physician is connected to most of the others and has access to the same amount and kind of knowledge. To capture closure, we modeled higher order transitivity (alternating $k$-triangles) and the preconditions for transitivity (alternating independent two-paths). In technical terms, the alternating $k$-triangle statistic represents triangulation in the network and moreover measures the extent to which triangles themselves group together in larger higher order 'clumps' (Robins et al. 2007, p. 200). It thus expresses transitivity as the tendency toward a comparatively high number of triangles, with the combination of alternating signs and decreasing weights of higher order $k$-triangles standing for an increase in the probability of observing a $k$-triangle which is a decreasing function of $k$. Alternating independent two-paths control for the prerequisites of triangulation, captured by the number of configurations that would be the sides of $k$-triangles if there existed a base edge (Snijders et al. 2006, p. 123). Hence, this corresponds to considering the effects of connections by two-paths, i.e. nodes $i$ and $j$ at a geodesic distance of two, regardless of whether or not the base of the triangle is present.

We then added to the model the exogenous effects, i.e. the individual and context-specific characteristics which are assumed to affect the propensity to engage in knowledge sharing (detailing, professional age, number of publications and hierarchical position). First, we considered the propensity of actors with specific values of the individual covariates to interact with others (first research question). These effects entered the model as main effects (Model 2). A positive parameter of this effect will imply the tendency that actors with higher values on one covariate have higher degree.

Second, we focused on the characteristics of the actors who are chosen as discussion partners (Model 3). More precisely, we examined the tendency of actors to interact with homophilous (i.e. similar) others. A positive parameter of the similarity effect implies that actors prefer to build ties to others with similar values on one covariate. We specify the similarity effect for either the sources of heterogeneity under exam or the control variables. These 
latter are specialty, affiliation and prominence. Similarity in specialty represents a tendency against multidisciplinary interaction. Similarity in affiliation captures geographic proximity, i.e. the propensity to discuss with geographically closed colleagues. It, thus, confutes the hypothesis of the existence of a wide network structure. Finally, similarity in prominence stands for inefficient interaction, since it means that one does not choose as discussion partners the most knowledgeable colleagues.

Homophily is modeled by dyadic covariates. Given a continuous actor covariate $x_{v}$, the corresponding dyadic one for the dyad $(i, j), w_{i j}$, is computed as the absolute difference $\left|x_{v i}-x_{v j}\right|$. For categorical actor covariates, $w_{i j}$ equals 1 if the two actors have the same value of the attribute and 0 otherwise. Dyadic covariates enter the model as a similarity effect for continuous variables and a same effect for categorical ones.

\subsubsection{Inference issues and goodness of fit}

The dataset used in this study was not a standard dataset for the application of ERGMs, because it was a sampled network. ERGMs, by contrast, have been designed for complete network data, i.e. they require the equivalent of a network census and assume that the presence or absence of all possible links is observed. These models, then, do not usually apply to sampled data, where the network is only partially observed. In this case, in fact, the inference is not straightforward. The MLEs from the sample could be biased, compared to complete data estimates, and some effects could be under- or over-estimated. In order to overcome these problems, Handcock and Gile (2010) have proposed methods which deal with sampled network data and attempt to model also the unobserved part of the network. To date, they have been applied to a small dataset ( $n=36$ actors). Because of the large network size, however, the methods in Handcock and Gile (2010) would be too labor-intensive here. We, then, decided to carry out the analysis with a regular ERG model, being very cautious in interpreting the results. We justify our choice referring to Snijders (2010). The author argues (cor. 3) that for saturated link-tracing samples (i.e. full connected components are sampled), and for models with terms with a local dependence property, it should be possible to fit an ERGM to the sampled data only. Our sample respects both these conditions and is, then, very close to a full-wave link-tracing sample of the kind required by Snijders (2010). Hence, it is likely reasonable to expect the parameters of the model fit to the sampled network to pick up on trends similar to those in the full network.

In regard to the ERGMs goodness of fit we followed two approaches. First, we verified whether the models converge for finite parameters values (Snijders 2002). To this purpose, the expected value and standard deviation of each statistic $u_{k}(y)$ were estimated from the generated networks and the $t$-ratios were then computed:

$$
t_{k}=\frac{\hat{E}_{\hat{\theta}}\left(u_{k}(Y)\right)-u_{0}}{\hat{S D_{\hat{\theta}}}\left(u_{k}(Y)\right)}
$$

The convergence is considered to be acceptable if $\left|t_{k}\right| \leq 0.2$.

Second, we computed the score-type test of single and multiple parameters implemented by Schweinberger (2008). This is a generalized Neyman's $C(\alpha)$ test and Rao's score test. The score-type test works as follows. It requires specifying a model with one or more parameters restricted to a constant (usually to 0 ). This restricted model is then estimated and a statistic test $c$ is calculated. $C$ tests the fit increase upon inclusion in the model of the restricted parameters. As the number of observations increases, the null distribution of $c$ tends to the chi-square distribution, with degrees of freedom equal to the number of restricted parameters. 


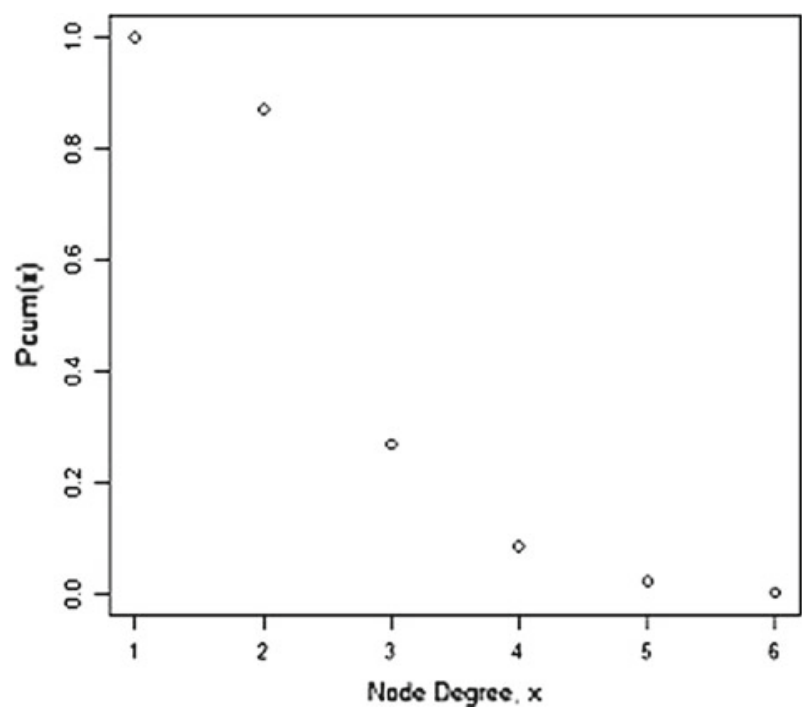

Fig. 1 Cumulative degree distribution

A high value of $c$ indicates a bad fit of the estimated model and suggests that the restricted parameters should be added to the model and estimated like the other parameters.

The score-type test is designed for the Method of Moments estimation. For the ML estimation (i.e. the ERGM case), it reduces to the Rao's efficient score test (Rao 1948). As stated above, we estimated several models, adding blocks of similar parameters one by one. We ran the generalized score-type test to verify the overall fit of each model. Then, with the separate score-type test, we observed the contribution of the single parameters and selected which ones to include. For brevity, the next section reports the three most relevant models. They verify our hypotheses and are also parsimonious.

\section{Results}

We begun the analysis by computing the main network descriptive statistics. The low value of density (0.003) suggests that the sampled network is extremely sparse. Then, only a very small proportion of the possible ties is formed. Figure 1 reports the cumulative degree distribution. Not surprisingly, it is positively skewed. The small degree range (max degree = 5 ), moreover, indicates that in the sampled network very central physicians are absent. Each individual interacts with few colleagues (average degree is 2.18), and there are not general opinion leaders. This result, however, could be biased by the sampling design. It, then, is to be interpreted very cautionary. We also observed that there is a significant percentage of isolates $(8.0 \%)$, i.e. seed nodes who had cited nobody as a discussion partner. Isolates are mainly research active (number of publications, mean $=1.203$ ) and experienced (years, mean $=29.673$ ) physicians.

Next step was the investigation of local network structures. To this purpose, we removed isolates and then examined the network components. As the network is undirected, we did not distinguish among weak and strong components. We found a high number of components, $207.43 \%$ of them are dyads or triads. The remaining $57 \%$ sized from 4 up to 12 . 
Table 2 ERGM estimates for the knowledge-sharing network

\begin{tabular}{lrrr}
\hline & Model 1 & Model 2 & \multicolumn{1}{c}{ Model 3} \\
\hline Structural effects & & & \\
Density & $-3.747(0.088)^{*}$ & $-3.845(0.223)^{*}$ & $-4.879(0.244)^{*}$ \\
Alternating k-stars & $-2.257(0.152)^{*}$ & $-1.817(0.218)^{*}$ & $-1.861(0.244)^{*}$ \\
Alternating k-triangles & $1.498(0.083)^{*}$ & $1.673(0.131)^{*}$ & $1.096(0.146)^{*}$ \\
Alternating independent two-paths & $0.432(0.106)^{*}$ & $0.056(0.126)$ & $0.090(0.152)$ \\
Main effects & & $-0.174(0.044)^{*}$ & $-0.215(0.052)^{*}$ \\
Professional age (experience) & & $-0.224(0.055)^{*}$ & $-0.379(0.140)^{*}$ \\
Publications (research orientation) & & $0.243(0.121)^{*}$ & $0.113(0.137)$ \\
Hierarchical position & & $0.015(0.019)$ & $0.061(0.022)^{*}$ \\
Detailing (external communication) & & & $-0.833(1.666)$ \\
Similarity effects & & & $-0.076(0.134)$ \\
Professional age (experience) & & & $1.995(0.465)^{*}$ \\
Publications (research orientation) & & & $6.695(0.147)^{*}$ \\
Hierarchical position & & & $0.806(0.171)^{*}$ \\
Detailing (external communication) & & & $-4.148(0.308)^{*}$ \\
Affiliation & & & \\
Specialty & & & \\
Prominence & & & \\
\hline & & & \\
\hline
\end{tabular}

Parameter values of the ERGM

Standard errors are in parentheses

$* p<0.05$ (two-sided test) $(n=784)$

The network is then highly disconnected and consists in a number of very small subnetworks, which only partially overlap. The smallest components (3-4 nodes) display a cliquelike structure. They are very dense, and, as expected, are centered within individual hospitals. By contrast, the largest components (7-12 nodes), which are a minority (6.0\%), are defined at national level and consist of very heterogeneous physicians. They are employed in different kinds of hospital organizations and are located in various Italian regions. In regard to individual characteristics, they do not significantly differ from the distribution in the sample.

Consistently with the literature, these findings suggest that, to date, knowledge-sharing relationships are largely based on proximity. Cooperation among distant physicians is not extensively exploited. Then, the high levels of geographic dispersion and uncertainty faced do not lead specialist physicians to look for higher competencies among distant colleagues.

We then turned to model knowledge sharing. The ERGMs were estimated using the SIENA program (Snijders 2005). Because the network is highly fragmented, we did not expect to observe very complex local structures. Therefore, in running the models we paid more attention to estimating the effects of the exogenous factors. Table 2 shows the results of the ERGMs, reporting the estimated parameters for each effect tested.

Because the models were built with a stepwise method, which includes a goodness-of-fit check, we consider the coefficients of Model 3 (the full model) to be the best estimates for the true value of the effects specified. We comment them in the same order as they appear in Sect. 3.2.2. 
First discussed is the block of structural parameters. The negative value for alternating $k$-star effect suggests a truncated degree distribution with a tendency against particularly high degree nodes. The physicians, then, are likely to discuss about the innovation and to share their knowledge with a small number of colleagues.

Next, we discuss the effect of the closure parameters, alternating $k$-triangles and alternating independent two-paths. While the latter is not significant in the full model, the positive effect of alternating $k$-triangles denotes a tendency toward triangulation. The combination of the alternating $k$-star with the high order transitivity parameters, moreover, highlights that the network tends to self-organize into several small groups of overlapping triangles (Robins et al. 2007). Consistently with previous studies on cooperation, physicians prefer to close in small teams of colleagues. This stands for lack of hierarchy within the group. Moreover, this result shows that there is not a strong opinion leadership, because each physician discusses with a similar number of colleagues and the network is not centralized around few individuals. Finally, closure indicates that physicians do not build relationships with colleagues outside their group. They, thus, do not have access to a large amount of new knowledge.

We examine, then, the role of individual and context-specific characteristics on tie formation. The results confirm our hypotheses, showing that physicians are heterogeneous in their propensity to share knowledge with others. As expected, the factors which affect the chance to adopt an innovation influence also the tendency to interact with colleagues. The strongest effect is that of research orientation (measured as the average number of publications per year). Being research productive, thus, decreases the propensity that a physician discusses and shares knowledge with his/her colleagues. Even more importantly, if we examine this effect from the opposite perspective, being poorly research productive increases the chance to rely on others as a source of information and influence. Because research orientation is likely to be correlated to interest in novelty and, hence, to propensity to innovate, the negative effect of this variable on knowledge sharing demonstrates that the latter is exploited mainly by physicians less keen to innovate. Therefore, it is perceived as a way to reduce the uncertainty of innovation adoption. Strong and negative is also the effect of experience. Because it is captured as professional age, a high value on this variable corresponds to seniority. The effect, then, implies that experienced and old physicians are likely to evaluate new products and services autonomously, while the younger have a higher propensity to discuss with their colleagues. Finally, still significant, but weaker, is the effect of external communication, i.e. detailing, on the tendency to build a tie. In contrary to what we expected, this effect is positive. A visit of detailing professionals increases by $\exp (0.061)=1.06$ times the chance to interact with others. More informed physicians, thus, have a higher propensity to engage in knowledge sharing. This finding indicates that external communication enhances, instead of replacing, knowledge sharing. Finally, the effect of the hierarchical position is not significant.

The third block of estimation results pertains to the similarity effects. They provide insights into the attributes of discussion partners. First, we analyze similarity in individual and contextual characteristics. The results show that the knowledge sharing network is shaped by similarity in experience and external communication. Both the effects are strong. Physicians, then, have a high propensity to engage in relationships with similarly aged and informed colleagues.

Then, we turn to examine the role of similarity in control variables, i.e. specialty and professional affiliation, and in prominence. The strongest is the effect of similarity in affiliation. Being in the same hospital significantly increases the probability to interact with colleagues. This finding confirms those of descriptive analysis. It is, moreover, intuitive and consistent with the literature. But it is also contrary to the expectations of NHS managers who promote and rely on interaction among geographically dispersed physicians, especially highly skilled 
ones. Weaker, but significant, is also the effect of similarity in specialty: physicians with the same scientific background are more likely to discuss on innovation. The odds of a tie between two physicians of a similar specialty are 2.24 times larger than for physicians in different specialties, conditioning on the rest of the network as well as on the other attribute effects.

Finally, it is apparent that knowledge sharing is shaped by the very strong effect of dissimilarity in prominence. Physicians, then, are more likely to discuss with knowledgeable colleagues. Because knowledge sharing is symmetric, it seems that the relationship with prominent colleagues is not hierarchical, like advice seeking. This result is consistent with the finding that knowledgeable physicians received a low number of nominations, and that, thus, their prominence is specific and local.

\section{Discussion and conclusions}

What drives individuals, physicians in particular, to rely on others as a source of information and influence on innovations? In order to answer this question, and also to provide better insights into the way knowledge flows among them, this paper analyzed knowledge sharing about a new drug in a large sample of specialist physicians. Our main goals were to understand whether physicians are heterogeneous in their propensity to interact with others and whether this heterogeneity is due to the same factors which affect the tendency to adopt innovations. Furthermore, we paid attention to how dyadic interaction combines into a network structure.

For these purposes, therefore, we addressed the issue from a social selection perspective and applied the Exponential Random Graphs (ERG) to model the probability of observing a tie between two physicians as conditional on attributes of the partners and on a variety of endogenous network sub-structures.

Before discussing the findings and the implications of this study, it is important to emphasize that we did not expert to provide an exhaustive overview on all the individual and context-specific characteristics which can affect one's choice to turn to colleagues as a source of information. Nor did we claim that interaction with others is the only factor leading physicians to take up a new product or service. However, its importance has been proven by a strong and very well established empirical evidence on diffusion of innovations.

Overall our analysis confirms that physicians are heterogeneous in the propensity to build ties with others. The descriptive network statistics point out the existence of isolate physicians, who do not interact with their colleagues, but also reveal that, let them aside, the most others are likely to have a similar and small number of discussion partners. Consistently with our hypothesis, the ERGMs estimates prove that this heterogeneity is mainly due to the same characteristics which influence the decision to adopt innovations. However, in some cases their effects on knowledge sharing are different from expectations. Moreover, the findings demonstrate that interaction consists in informal and mutual relationships of discussion or knowledge sharing. These relationships develop within small and closed communities of peers, shaped by similar attitudes and geographic proximity.

The analyses were performed at three different levels. We discuss their results in the same order as they appear in the previous section. First, at network level, we found that physicians display a high propensity to reciprocity and closure. They tend to share information and knowledge informally with their colleagues, instead of specifically going to them for advice seeking. This relationship is based on mutual trust and is not strictly hierarchical. Because this result is consistent with the findings of organizational studies (Keating et al. 2007) more than with those on diffusion of innovations, we conjecture that highly skilled physicians 
perceive the decision to adopt an innovation as a cooperative one. The tendency to closure, moreover, indicates that each physician is likely to dispose of similar information. Hence, there is neither information asymmetry nor access to a great amount of new, fresh knowledge.

At individual level, it is apparent that the propensity to engage in knowledge sharing is enhanced by a negative attitude toward innovation. Less knowledgeable (lack of experience) and more risk averse (lack of research orientation) physicians are more ready to interact with others. In regard to experience, it is worth to recall that low values on this variable correspond also to low tenure. Hence, being younger and less experienced increases the tendency to discuss with others (Barlow and Burn 2008). Contrarily to expectations, detailing (which stands for external communication) is not perceived as alternative to knowledge sharing. It therefore positively affects the choice to build ties. More informed physicians display a higher propensity to share their opinions and to turn to others to be confirmed in (or modify) their own beliefs. By contrast, their colleagues less subject to detailing are more likely to remain isolated and scarcely informed. As a consequence, they are also less likely to take up innovations.

Finally, at dyadic level, we find that the network is shaped by similarity in attitudes toward innovation. This is expected to positively affect physicians with a positive attitude, and negatively the others. Knowledge sharing with similar colleagues leads experienced and research oriented physicians (in other words risk seekers) to acquire relevant opinions on the new drug, while interaction among less experienced and keen to innovate actors is likely to be poorly informative, therefore also useless and ineffective. Most interaction takes place within the hospital organization, as expected, and among physicians in the same specialty. Only few actors are likely to build ties to distant colleagues. In this preference for local knowledge sharing, then, specialist physicians do not significantly differ from the other doctors. The high level of uncertainty that oncologists and anesthetists have to deal with does not drive them to look for the most expert colleagues in the field. This behavior could be explained by dissimilarity in prominence. The most physicians display a high tendency to discuss and share knowledge with colleagues that they consider prominent. Because the most interaction takes place within the hospital, prominence, and consequently opinion leadership, are very local (Valente and Davis 1999; Clark et al. 2010). Given the cost to establishing new relationships with nationally reputed experts, relying on local and preexisting ties seems to be the optimal strategy in terms of net benefit for markets in which physicians are usually overburdened with information, like the drug market.

The main conclusion of this study is that interaction can be a powerful tool for the diffusion of innovations, but needs to be generally and massively sustained by other strategies, mainly by communication campaigns. In fact, only physicians with very specific characteristics tend to share information and beliefs with others. Furthermore, this tendency is promoted by the exposure to other sources of information. Also, because of closure in hospital-centered small sub-networks, knowledge transmission is unlikely to lead to a general and quick convergence of behavior on innovations. Influence then can be exerted only within each group.

This paper, of course, have limitations. First, it is a case study on a sampled nationwide network. This sampling design was chosen in order to take account of very different work environments, whose characteristics (hospital size, interest and investments in research advancements, public funding received) were not explicitly framed in our study, but deserve attention. Furthermore, by fixing the boundaries at a national level, we were able to verify whether specialist and high skilled physicians display a significant propensity to build ties with distant colleagues. Nevertheless, this design could have affected the results in two ways. Because of the wide boundaries, we had to deal with a sparse and disconnected network, consisting in several small sub-networks centered within individual hospitals and in few 
larger components, which included very geographically dispersed physicians. The findings on the local network effects are then to be very cautionary interpreted. Moreover, as we highlighted when discussing inference issues, estimates of an ERG model from a sampled network are likely to be down-ward biased. In this respect, however, there are insights that in our study these effects were moderate. Also, our results on the network structure are perfectly consistent with the literature (Coleman et al. 1966; Keating et al. 2007; West et al. 1999).

A second limitation is represented by the proxies that had to be defined in order to measure some variables not directly observable, e.g. experience in the field and research orientation. We justified our choices, linking these proxies to previous studies. Moreover we tried to verify their consistency with the corresponding variables (in respect to research orientation, the number of publications was chosen as a proxy after indepth interviews with a small sample of oncologists). However, in the future a more accurate operationalization could be tested.

Future work will focus, furthermore, on extending our research to different settings, in order to encompass and explicitly model various levels of uncertainty. Also, we will strive to include in this framework the effects of other individual and context-specific variables, which could not be collected here.

\section{References}

Barlow, J., Burn, J.: All Change Please. Policy Exchange, London (2008)

Burt, S.: Social contagion and innovation: cohesion versus structural equivalence. Am. J. Sociol. 92(6), 12871335 (1987)

Burt, R., Janicik, G. : Social contagion and social structure. In: Iacobucci, D. (ed.) Networks in Marketing, pp. 68-89. Sage, Thousand Oaks (1996)

Clark, M., Linkletter, C., Wen, X., Miller, E., Mor, V.: Opinion networks among long-term care specialists. Med. Care Res. Rev. 67(4), 102-125 (2010)

Cohen, W., Levinthal, D.: Absorptive capacity: a new perspective on learning and innovation. Adm. Sci. Q. 35, 128-152 (1990)

Coleman, S., Katz, E., Menzel, H.: Medical Innovation: A Diffusion Study. The Bobbs-Merrill Company, Indianapolis (1966)

Coscelli, A., Shum, M.: An empirical model of learning and patient spillovers in new drug entry. J. Econom. 122, 213-246 (2004)

Creswick, N., Westbrook, J.: Examining the socialising and problem-solving networks of clinicians on a hospital ward, working paper series. University of New South Wales (2006)

Doreian, P., Woodard, K.: Defining and locating cores and boundaries in social networks. Soc. Netw. 16, 267293 (1994)

Fattore, G., Salvatore, D.: Network organizations of general practitioners: antecedents of formation and consequences of participation. BMC Health Serv. Res. 10(1), 118-129 (2010)

Fennell, M., Warnecke, R.: The Diffusion of Medical Innovations: An Applied Network Analysis. Plenum, New York (1988)

Gollop, R., Whitby, E., Buchanan, D., Ketley, D.: Influencing sceptical staff to become supporters of service improvement: a qualitative study of doctors and 'managers' views. Qual. Saf. Health Care 13, 108114 (2004)

Handcock, M., Gile, K.: Modeling social networks from sampled data. Ann. Appl. Stat. 4(1), 5-25 (2010)

Hirschman, E.: Innovativeness, novelty seeking, and consumer creativity. J. Consum. Res. 7(3), 283-295 (1980)

Kaluzny, A., Ricketts, T., Warnecke, R., Ford, L., Morrissey, J., Gillings, D., Sondik, E., Ozer, H., Goldman, J.: Evaluating organizational design to assure technology transfer: the case of the community clinical oncology program. J. Natl. Cancer Inst. 81, 1717-1725 (1989)

Katz, E., Lazarsfeld, P.: Personal Influence. Free Press, Glencoe (1955)

Keating, N., Ayanian, J., Cleary, P., Marsden, P.: Factors affecting influential discussions among physicians: a social network analysis of a primary care practice. J. Gen. Intern. Med. 22(6), 794-798 (2007)

Lave, J., Wenger, E.: Situated Learning: Legitimate Peripheral Participation. Cambridge University Press, Cambridge (1991) 
Mukherjee, P.: A structural model of learning for new products, working paper series. University of Minnesota (2002)

Nair, H., Manchanda, P., Bhatia, T.: Asymmetric social interactions in physician prescription behavior: the role of opinion leaders, working paper series. Stanford University (2008)

Pattison, P., Robins, G.: Neighbourhood based models for social networks. Sociol. Methodol. 22, 301337 (2002)

Rao, C.: Large sample tests of statistical hypotheses concerning several parameters with applications to problems of estimation. Proc. Camb. Philos. Soc. 44, 50-57 (1948)

Robins, G., Elliott, P., Pattison, P.: Network models for social selection processes. Soc. Netw. 23, 1-30 (2001)

Robins, G., Snijders, T., Wang, P., Handcock, M., Pattison, P.: Recent developments in exponential random graph $\left(p^{*}\right)$ models for social networks. Soc. Netw. 29, 192-215 (2007)

Schweinberger, M.: Statistical modeling of network dynamics given panel data: goodness-of-fit tests (2008, submitted for publication)

Snijders, T.: Markov chain Monte Carlo estimation of exponential random graph models. J. Soc. Struct. 3(2), $1-40$ (2002)

Snijders, T: Models for longitudinal network data. In: Carrington, P., Scott, J., Wasserman, S. (eds.) Models and Methods in Social Network Analysis, pp. 215-247. Cambridge University Press, Cambridge (2005)

Snijders, T.: Conditional marginalization for exponential random graph models. J. Math. Soc. 34(4), 239-252 (2010)

Snijders, T., Pattison, P., Robins, G., Handcock, M.: New specifications for exponential random graph models. Sociol. Methodol. 36, 99-153 (2006)

Strang, D., Tuma, N.: Spatial and temporal heterogeneity in diffusion. Am. J. Sociol. 99, 614-639 (1993)

Trzeciak, R., Dellinger, P., Abate, N., Cowan, R., Stauss, M., Kilgannon, J., Zanotti, S., Parrillo, J.: Translating research to clinical practice. Chest 129, 225-232 (2006)

Valente, T.: Social network thresholds in the diffusion of innovations. Soc. Netw. 18, 69-89 (1996)

Valente, T., Davis, R.: Accelerating the diffusion of innovations using opinion leaders. Ann. Am. Acad. Political Soc. Sci. 566(1), 55-67 (1999)

Valente, T., Pumpuang, P.: Identifying opinion leaders to promote behavior change. Health Educ. Behav. 34(6), 881-896 (2007)

Van den Bulte, C., Joshi, Y.: New product diffusion with influentials and imitators, working paper series. The Wharton School, University of Pennsylvania (2006)

Van den Bulte, C., Lilien, G.: Medical innovation revisited: social contagion versus marketing effort. Am. J. Sociol. 106(5), 1409-1435 (2001)

Wasserman, S., Pattison, E.: Logit models and logistic regression for social networks: I. An introduction to Markov graphs and $p^{*}$. Psychometrika 61, 401-425 (1996)

West, E., Barron, D., Dowsett, J., Newton, J.: Hierarchies and cliques in the social networks of health care professionals: implications for the design of dissemination strategies. Soc. Sci. Med. 48(5), 633-646 (1999)

Zappa, P., Mariani, P.: The interplay of social interaction, individual characteristics and external influence in diffusion of innovation processes: an empirical test in medical settings. Procedia Soc. Behav. Sci. 4th \& 5th UK Social Networks Conferences 10, 140-147 (2011) 
Reproduced with permission of the copyright owner. Further reproduction prohibited without permission. 